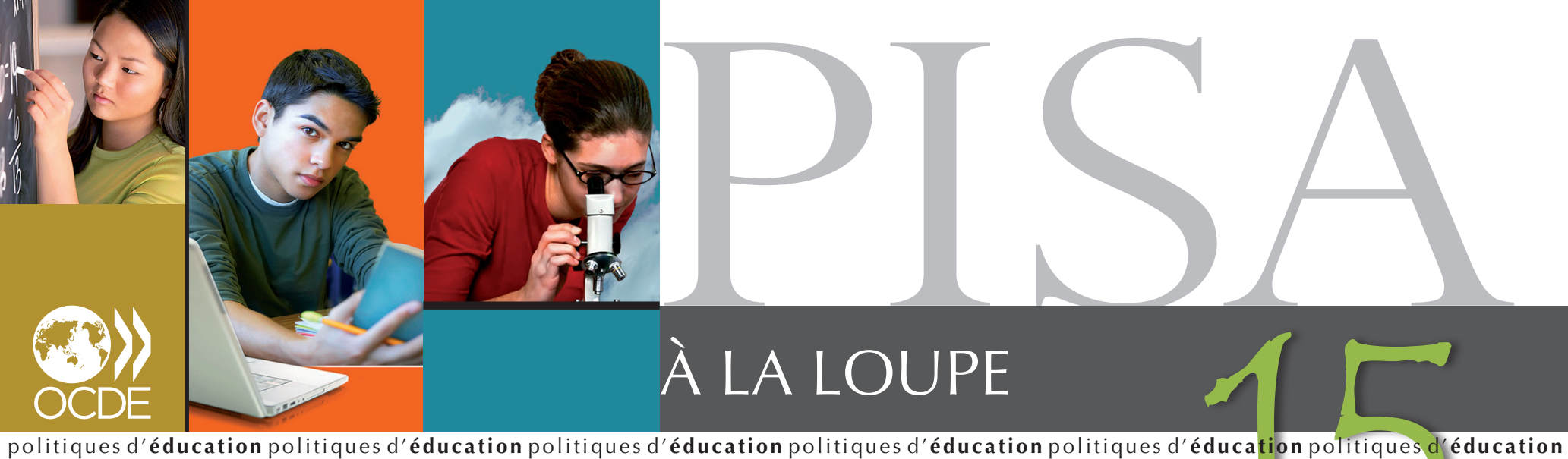

\title{
Aujourd'hui, les jeunes de 15 ans sont-ils « verts »?
}

- Dans les pays de l'OCDE, environ un élève sur cing est en mesure d'identifier, d'expliquer et d'appliquer des concepts scientifiques liés à différentes thématiques environnementales. Au Canada, en Finlande et au Japon, plus d'un tiers des élèves de 15 ans font preuve d'une bonne connaissance des problèmes environnementaux.

- Les élèves acquièrent la majorité de leurs connaissances sur l'environnement à l'école, bien que seule une minorité d'entre eux bénéficient de cours entièrement dédiés aux sciences de l'environnement.

- Pour les élèves, l'école constitue une source essentielle d’informations sur les thématiques environnementales. Alors que dans la plupart des pays, seule une minorité des établissements d'enseignement proposent des cours spécifiquement consacrés à l'environnement, ce sujet est souvent abordé dans le cadre d'autres matières principales du programme, et de nombreux établissements organisent des activités sur le thème de l'environnement en dehors des salles de classe.

Dans certains pays, une forte proportion d'élèves sont bien informés sur les problèmes environnementaux..

De nos jours, l'environnement naturel dans lequel grandissent les élèves est précaire. Le changement climatique et l'appauvrissement de la biodiversité menacent les écosystèmes nécessaires à la vie ; le manque d'eau salubre et de réseaux $d^{\prime}$ assainissement constitue un risque sanitaire quotidien pour des centaines de millions d'individus. Si les géoscientifiques, les biologistes et les spécialistes de l'environnement occupent, forts de leur expertise, une place privilégiée dans l'élaboration des stratégies visant à réduire l'impact de l'activité humaine sur l'environnement mondial - et à garantir un accès plus équitable aux ressources naturelles pour tous - les citoyens informés jouent, eux aussi, un rôle important. Au vu de l'impact des actions individuelles sur l'environnement, la compréhension des théories scientifiques et la capacité d'interpréter des données peuvent permettre à tout un chacun de faire des choix éclairés sur des questions aussi quotidiennes que laisser ou non son téléviseur en veille, régler son chauffage à la bonne température et choisir le type de voiture à acheter (ou non). Une sensibilisation précoce des élèves à l'environnement dans leur parcours scolaire peut influer sur la façon dont ils interagiront avec l'environnement à l'âge adulte.

Les élèves de 15 ans sont-ils « verts » ? Dans quelle mesure comprennent-ils les problèmes environnementaux ? Quelles sont les sources d'informations dont ils disposent à ce sujet? Et sont-ils capables d'appliquer des principes et des données scientifiques au monde qui les entoure? 
En moyenne, dans les pays de l'OCDE, 19 \% des élèves se classent au plus haut niveau de l'échelle PISA de compétences en sciences de l'environnement (niveau A). Les élèves atteignant ce niveau sont capables d'identifier, d'expliquer et d'appliquer des connaissances scientifiques liées à différentes thématiques environnementales. Ils sont en mesure d'établir des liens entre différentes sources d'information et explications, et d'y puiser des éléments pertinents pour justifier des décisions relatives aux thématiques environnementales. Ils sont systématiquement capables de se livrer à des réflexions et à des raisonnements scientifiques approfondis sur des thèmes liés à l'environnement. Ils parviennent à exploiter ces connaissances pour développer des arguments en faveur de recommandations ou de décisions dans des situations sociales ou mondiales. Ces élèves les plus performants représentent un vivier potentiel de citoyens bien informés, compétents et dotés d'un bon esprit d'analyse susceptibles de devenir les scientifiques et les spécialistes de l'innovation de demain.

Parmi les pays de l'OCDE, le Canada, I'Estonie, la Finlande, le Japon, la Corée et la Slovénie présentent des proportions particulièrement importantes d'élèves se classant au plus haut niveau de l'échelle PISA de compétences en sciences de I'environnement. À quelques rares exceptions près, entre $15 \%$ et $31 \%$ des élèves des pays de l'OCDE atteignent ce niveau.

Pourcentage d'élèves à chaque niveau de compétences de l'échelle PISA de performance en sciences de l'environnement

$\%$

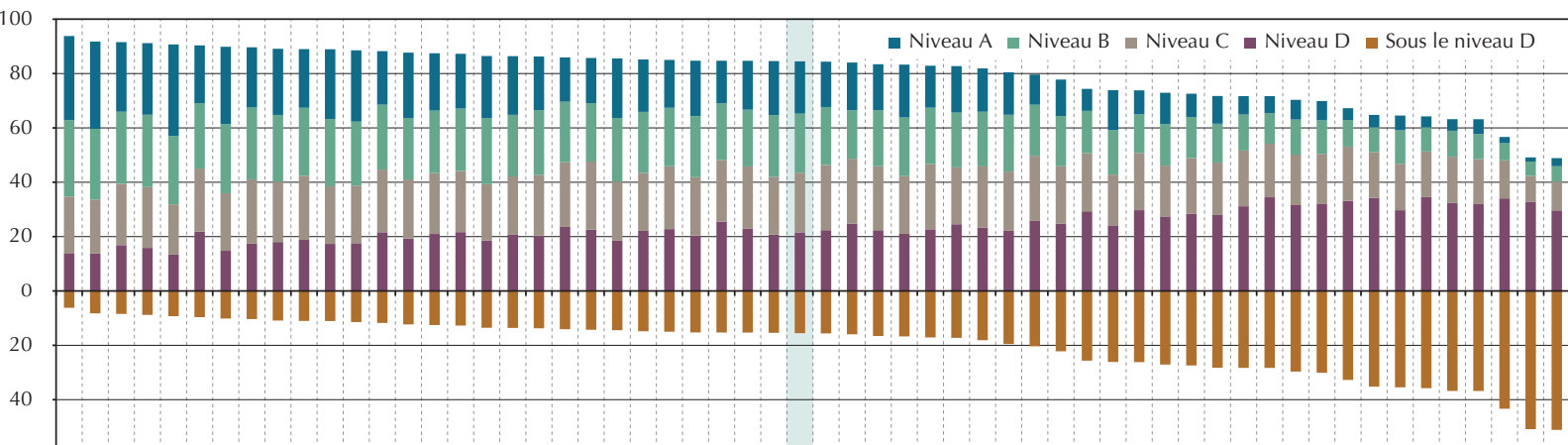

60

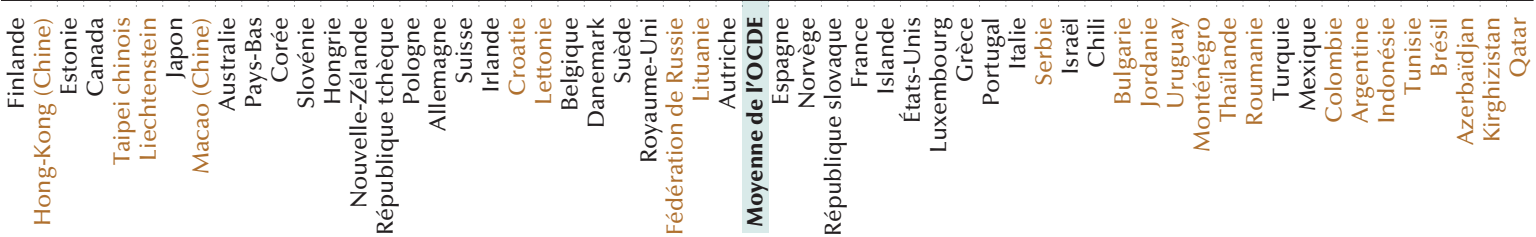

Les élèves atteignant le niveau A de compétences sont capables d'identifier, d'expliquer et d'appliquer des connaissances scientifiques liées à différentes thématiques environnementales. Ils sont en mesure d'établir des liens entre différentes sources d'information et explications, et d'y puiser des éléments pertinents pour justifier des décisions relatives aux thématiques environnementales. Les élèves se situant sous le niveau $D$ de compétences ne sont pas capables de répondre à des questions comprenant des informations scientifiques en rapport avec des phénomènes ou des problèmes environnementaux élémentaires.

Remarque : les pays et les économies sont classés par ordre croissant du pourcentage d'élèves de 15 ans se situant sous le niveau D de compétences. Source : Base de données PISA 2006 de l'OCDE.

. mais dans d'autres pays, une proportion importante d'élèves sont mal équipés pour faire face aux défis environnementaux.

À l'autre extrémité de l'échelle de compétences, un pourcentage significatif d'élèves ne sont pas capables de répondre à des questions comprenant des informations scientifiques en rapport avec des phénomènes ou des problèmes environnementaux élémentaires. Ces élèves affichent une performance inférieure au niveau seuil sur l'échelle PISA de compétences en sciences de l'environnement (soit le niveau D). La proportion d'élèves de 15 ans peu performants en sciences de l'environnement est un indicateur important de la disponibilité dans un pays d'une population adulte bénéficiant d'un degré suffisant de connaissances et de compréhension pour faire face aux défis environnementaux de demain. En moyenne, dans les pays de l'OCDE, 16 \% des élèves se classent en deçà de ce niveau seuil de compétences; dans quatre pays de l'OCDE, 20 \% des élèves, voire davantage, sont dans ce cas. Au Kirghizistan et au Qatar, c'est plus de la moitié des élèves qui n'atteignent pas le niveau seuil de compétences. Par contraste, au Canada, en Estonie, en Finlande, au Japon et, dans les pays et économies partenaires, au Liechtenstein, au Taipei chinois, à Hong-Kong (Chine) et à Macao (Chine), 10 \% des élèves, et parfois moins, sont concernés. 
Comment les sciences de l'environnement sont-elles enseignées à l'école ? La quasi-totalité des élèves des pays de I'OCDE fréquentent des établissements qui intègrent l'enseignement des sciences de I'environnement aux autres matières scientifiques du programme; ce constat n'est pas valable pour seulement $2 \%$ des élèves de l'OCDE, en moyenne. La Grèce et le Japon font figure d'exception notable : selon les chefs d'établissements, seuls $15 \%$ et $13 \%$ des élèves, respectivement, y fréquentent des établissements qui n'enseignent pas les sciences de l'environnement dans le cadre du programme de sciences. Parmi les différents pays, seule une minorité d'élèves fréquentent des établissements où l'enseignement des thématiques environnementales se déroule dans le cadre de cours consacrés à part entière à ce sujet. La plupart des élèves acquièrent leurs connaissances en sciences de l'environnement lors des cours de matières connexes, telles que les sciences naturelles ou la géographie.

\section{Lorsqu'il s'agit de l'environnement, les méthodes d'enseignement et d'apprentissage sont souvent} novatrices.

La sensibilisation aux thématiques environnementales intervient de plus en plus en dehors des salles de classe. Selon les chefs d'établissement, la plupart des élèves de 15 ans fréquentent des établissements qui organisent au moins une activité d'apprentissage à l'extérieur de leur enceinte. Parmi les activités proposées, les classes de plein air sont les plus courantes, suivies des visites de musées et de centres scientifiques. En moyenne, les élèves des pays de l'OCDE fréquentent des établissements d'enseignement qui proposent : des classes de plein air (77\% des élèves) ; des visites de musées (75\% des élèves) ; et des visites de centres scientifiques $(67 \%$ des élèves). Toutefois, l'offre de ce type d'activités varie fortement selon les pays : au Japon, par exemple, $55 \%$ des élèves fréquentent des établissements d'enseignement qui, selon leurs chefs d'établissements, ne proposent aucune de ces activités hors du cadre scolaire, tandis qu'au Portugal et en République slovaque, tous les établissements d'enseignement proposent au moins l'une de ces activités.
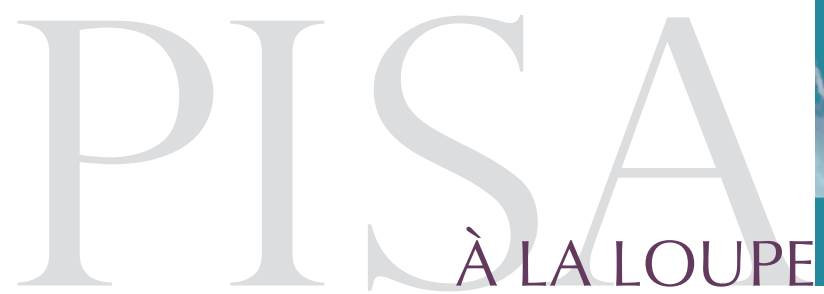

\section{L'environnement en classe}

Les élèves sensibilisés à l'école à des sujets tels que l'extinction de certaines espèces animales et végétales obtiennent de meilleurs résultats à l'évaluation PISA des sciences de l'environnement.

\section{Écart en points de score PISA}

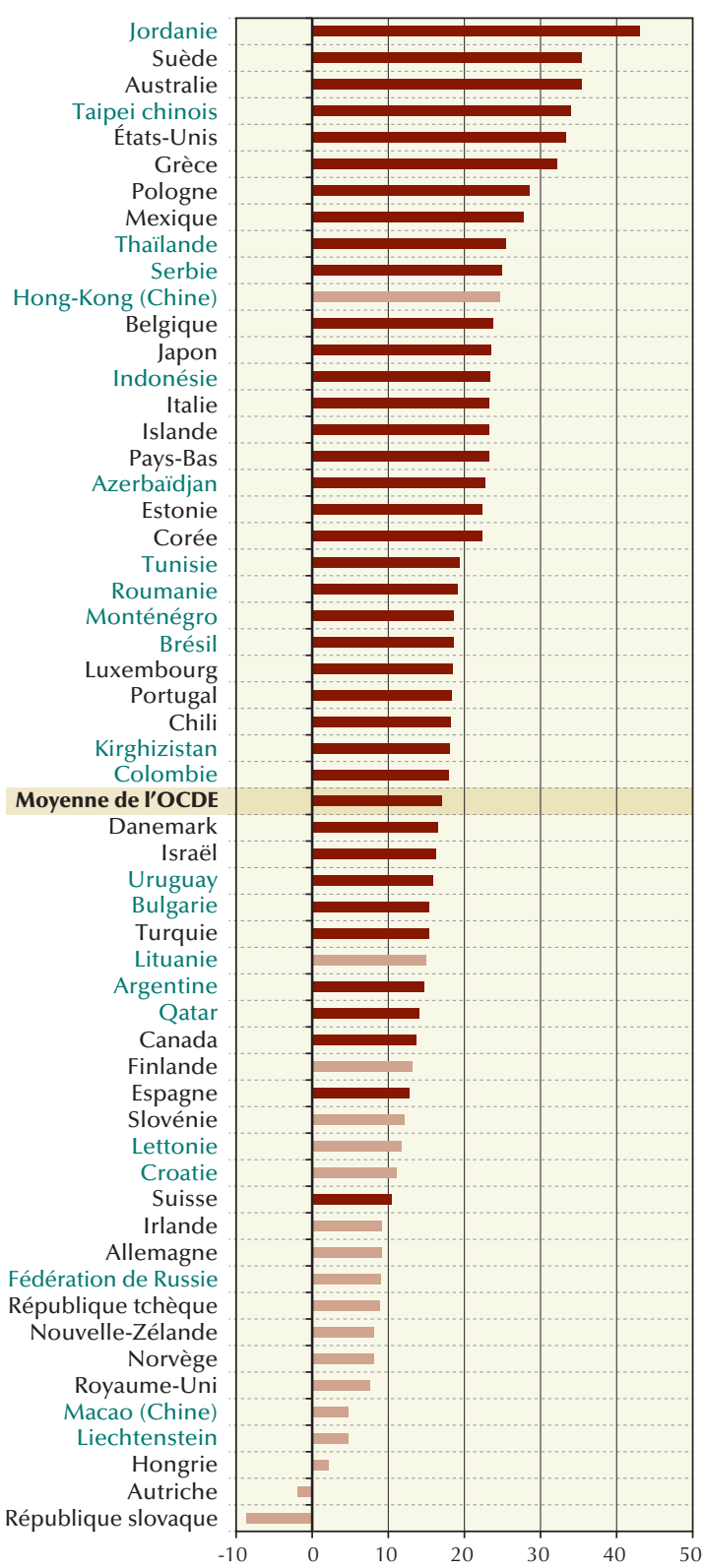

Remarque : les écarts en points de score tiennent compte des variables contextuelles. Les valeurs statistiquement significatives sont indiquées en couleur plus foncée.

Source : Base de données PISA 2006 de l'OCDE. 
Les établissements d'enseignement jouent un rôle primordial dans la sensibilisation des élèves à des thématiques environnementales aussi essentielles que la pollution atmosphérique, l'énergie, l'extinction de certaines espèces animales et végétales, la déforestation, les pénuries d'eau et les déchets nucléaires. C'est à l'école que les élèves prennent le plus souvent connaissance de ces sujets. Les élèves plus performants utilisent également les médias et Internet pour élargir et approfondir leurs connaissances. En développant les compétences des élèves en sciences de l'environnement et en incluant l'environnement dans les différentes parties du programme scolaire, l'école peut susciter un intérêt pour ce sujet qui perdurera en dehors de son enceinte et jusqu'à l'âge adulte.

Quelles sont les différentes sources d'information des élèves ?
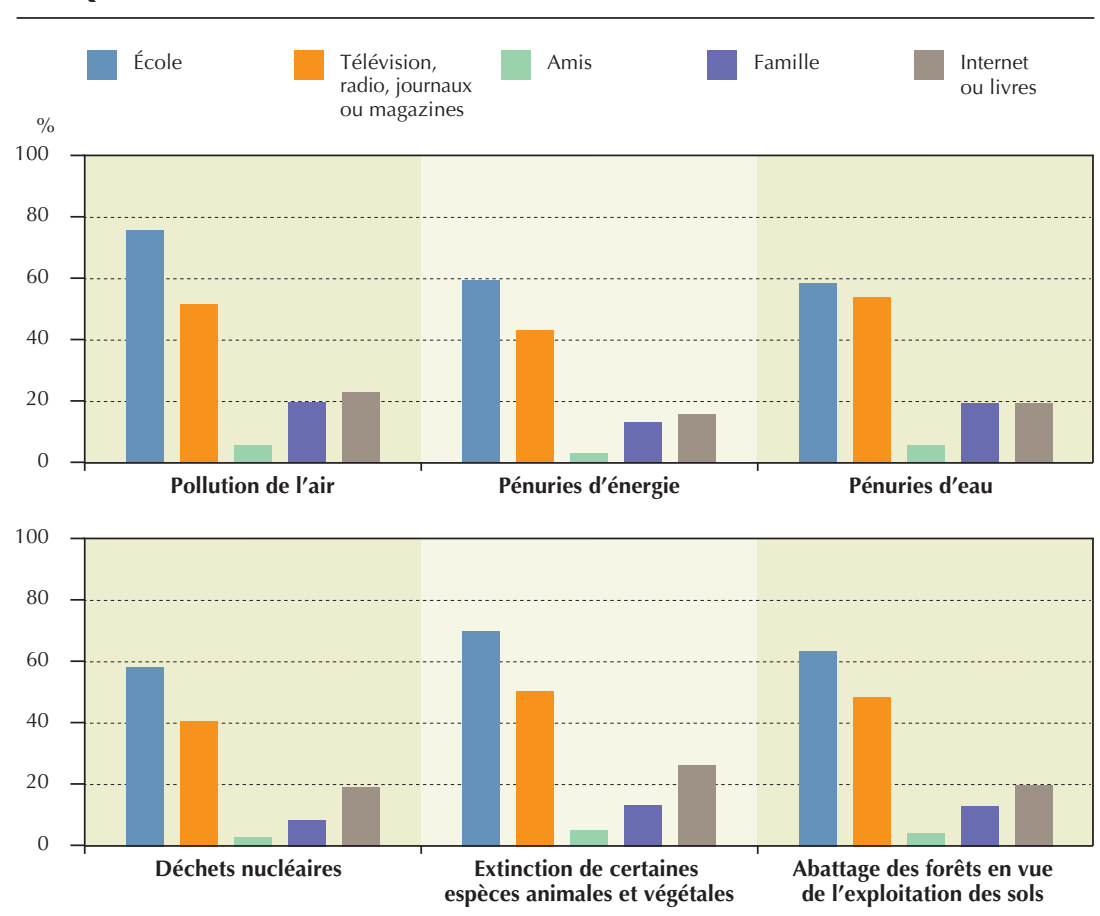

Source: Base de données PISA 2006 de l'OCDE.

Pour conclure: Les actions des individus ont un impact sur l'environnement. Les élèves qui sont sensibilisés aux questions environnementales à l'école sont mieux informés à ce sujet et cherchent aussi, en général, à diversifier leurs sources d’informations sur l'environnement. Équipés de ces connaissances, les élèves sont mieux préparés à faire face aux choix difficiles qui se présenteront à eux une fois parvenus à l'âge adulte dans un environnement naturel de plus en plus menacé.

\section{Pour tout complément d'information}

Contacter Francesca Borgonovi (Francesca.Borgonovi@oecd.org)

Consulter OCDE (2009), Green at Fifteen? How 15-year-olds Perform in Environmental Science and Geoscience in PISA 2006, Éditions OCDE (disponible en anglais uniquement).

Voir

www.pisa.oecd.org

www.oecd.org/pisa/infocus
À paraître ce mois-ci, un numéro spécial :

Connaissances et compétences: des richesses plus précieuses que le pétrole, car inépuisables 